American Journal of Pharmacology and Toxicology 1 (4): 72-78, 2006

ISSN 1557-4962

(C) 2006 Science Publications

\title{
The Phenotypically Suppressed Cancer Cell As a Therapeutic Target
}

\author{
${ }^{1}$ Michael A. Ihnat, ${ }^{1}$ Kimberly D. Kyker, ${ }^{1}$ Jessica E. Thorpe \\ ${ }^{1,2}$ Satyendra S. Shenoy and ${ }^{1}$ Robert E. Hurst \\ ${ }^{1}$ Departments of Urology, Cell Biology and Biochemistry and Molecular Biology \\ University of Oklahoma Health Sciences Center, Oklahoma City, OK 73190 \\ ${ }^{2}$ Department of Pharmacology, Nicholas Piramal Research Centre, Mumbai, India
}

\begin{abstract}
Bladder cancer recurs in up to $70 \%$ of patients judged to be tumor-free after treatment. Like other cancers, delayed appearance of metastases is common. These findings suggest the malignant phenotype can be suppressed, to emerge later. Following up on previous research in our laboratory showing that normal extracellular matrix can suppress the malignant phenotype of bladder cancers, we here demonstrate that the suppression of the malignant phenotype in culture models of bladder cancer cells grown on a gel derived from normal extracellular matrix also occurs in flank xenograft models. In addition, we also demonstrate a novel screening method to identify drugs that preferentially target suppressed bladder cancer cells that normally are resistant to conventional chemotherapeutic agents. This screening method could potentially identify completely novel therapeutic agents targeting the suppressed cancer cell and could be used to prevent recurrence and destroy potentially fatal micrometastatic tumors.
\end{abstract}

Key words: Dormant tumor, bladder carcinoma, extracellular matrix, suppressed phenotype

\section{INTRODUCTION}

About 60,000 new cases of bladder cancer occur each year in the U.S. with about 13,000 deaths, placing it $5^{\text {th }}$ overall in cancer incidence ${ }^{[1]}$. In the United States, $98 \%$ of bladder cancers arise from the transitional epithelium of the bladder (transitional cell carcinoma, TCC ${ }^{[2]}$. The general perception that bladder cancer is not serious is false. Some $15-25 \%$ of cases are invasive at diagnosis with one-fourth already having metastasized and with up to half developing metastatic tumors within 2-3 years ${ }^{[3,4]}$. The 5 -year survival of patients with metastatic bladder cancer is very low, about $20 \%$ with about 6 month median survival for even the most aggressive therapies ${ }^{[5,6]}$. Of the $80-85 \%$ that are papillary, recurrence is high, up to $70 \%$ within 5 years in some studies and of these, some $15 \%-25 \%$ will progress to invasive bladder cancer ${ }^{[3]}$. Therapy achieves few cures, whether with $\mathrm{BCG}^{[3,7]}$ chemotherapy ${ }^{[5,6]}$ or neoadjuvant chemotherapy ${ }^{[8,9]}$ The reasons for the high recurrence rate of bladder cancer are not known entirely. Three mechanisms are suggested; underdiagnosis by cystoscopy ${ }^{[10]}$, a widespread "field defect" with continued promotion of new tumors or suppression of malignant cells, preventing their growth for a time ${ }^{[11]}$.

The evidence for the suppression of malignant properties is particularly interesting because it suggests an important, but heretofore ignored, phenomenon that could affect recurrence. Very compelling evidence for suppression of the malignant phenotype is the presence of identical microsatellite mutations in the primary tumor and in the distant, pathologically normal urothelium $^{[12,13]}$, the finding of aberrant biomarkers up to $10 \mathrm{~cm}$ distant from the tumor in histopathologically normal epithelium ${ }^{[14]}$ and deletions from chromosomes $9 \mathrm{p}^{[15]}$ as well as of $8 \mathrm{p}^{[16]}$.

We previously described a model for investigating the phenomenon of suppression of the malignant phenotype ${ }^{[17,18]}$. In this model, cancer cells grown on a cancer-modulated extracellular matrix (ECM) material (Matrigel) fully express the malignant phenotype but on a normal ECM derived material (SISgel, small intestine submucosa) the malignant phenotype is suppressed. We first demonstrate that suppression also occurs in vivo in the flank xenograft model. Virtually the entire multibillion dollar anticancer therapeutic industry is based on preclinical screening involving cancer cells grown on

Corresponding Author: $\quad$ Robert E. Hurst, Department of Urology, Oklahoma University Health Sciences Center, 107, BMSB, 940 S.L. Young Blvd, Oklahoma City, OK 73104, Tel: 405-271-3930, Fax: 405-271-3289 
plastic in conventional tissue culture. Previously, we showed that bladder cancer cells grown on either Matrigel of SISgel were much more resistant to common bladder cancer therapeutics than when grown on plastic ${ }^{[19]}$. We herein describe a novel screening method to identify lead compounds based on finding preferential activity against cells grown on SISgel than on plastic. The finding that such compounds exist suggests a completely novel set of anticancer drugs that specifically target suppressed cells responsible for occult metastasis and recurrence could potentially be identified.

\section{MATERIALS AND METHODS}

Cell culture: All tissue culture media and supplements were from Invitrogen, Rockville, MD. Matrigel was obtained from Becton-Dickinson (Bedford, MA). SISgel was obtained from Cook Biotech (W. Lafayette, IN). TCC-SUP, J82 and 5637 cells were obtained from the American Type Culture Collection (ATCC).

Flank xenograft model: J82 cells stably expressing green fluorescent protein (GFP) were prepared as follows. pLEGFP-C1 retrovirus (Clontech, Mountain View, CA) was co-transfected with the pVSV-G vector (Clontech, Mountain View, CA) which contains a viral envelope gene into the packaging cell line GP2-293 (Clontech, Mountain View, CA). Supernatant from the packaging cells containing the infective virus was collected every 24 hours for 4 days. Fluorescent target cells were made by infecting J82s, a urothelial transitional cell carcinoma cell line (ATCC, Manassas, VA), with $1 \mathrm{~mL}$ of fresh, virus containing, supernatant/well containing 100,000 target cells along with $8 \mu \mathrm{g} \mathrm{mL}^{-1}$ of polybrene (Sigma Aldrich, St. Louis, MO). Each application of viral supernatant was filtered through a $0.4 \mu \mathrm{M}$ syringe filter before application to target cells. Supernatant was removed and fresh virus containing media replenished on target cells every 24 hours until 4 changes of media were completed. Virus containing media was replaced with Minimum Essential Media, MEM, (Life Technologies, Carlsbad, CA) containing $1 \%$ nonessential amino acids, $1 \%$ Lglutamine, $1 \%$ sodium pyruvate and 10\% Fetal Calf Serum and cells were allowed to grow to $90 \%$ confluence. Stable transfects were selected through sequential sorting and enrichment of fluorescent cells using flow cytometry.

In sterile $500 \mu \mathrm{L}$ centrifuge tubes, placed on ice, $100 \mu \mathrm{L}$ of cell suspension was mixed with either $100 \mu \mathrm{L}$ ice-cold prepared SISgel or ice-cold Matrigel and mixed well. The mix was immediately injected into either the right or left flank of a 5-week old nude mouse, nu/nu-nuBR, (Charles River Laboratories, Wilmington, MA). Caliper measurements of tumor size were taken every week for the length of the study as were fluorescent images. Images were visualized with the Lightool's LT-9900 system (Lightools, Encinitas, CA) with the EGFP filter set of 470nm excitation filter and $515 \mathrm{~nm}$ viewing filter and captured with a Nikon DC290 digital camera. The area and intensity of the tumor was measured using Adobe Photoshop by first selecting the tumor area, then counting pixels above a threshold selected to eliminate background, non-tumor areas. The integrated intensity was calculated by multiplying the average intensity of detected pixels by the number of pixels detected.

Plating of cells for screening: Three-dimensional gel cultures with Matrigel were made by layering $50 \mu \mathrm{L}$ of ice cold Matrigel into wells of a Costar 3610 white, clear bottom 96-well plates (Corning, Corning, NY) allowed to gel at $37^{\circ} \mathrm{C}$. Confluent cells were trypsinized with $1 \mathrm{~mL} \quad 0.25 \%$ trypsin $-1 \mathrm{mM}$ EDTA and 30,000 were added to wells containing the gelled matrices. The cells were fed with $50 \mu \mathrm{L}$ of their respective media containing $10 \%$ fetal calf serum. To establish non-confluent monolayers, cells were plated at a cell density of $10,000 /$ well $24 \mathrm{~h}$ prior to drug treatments.

Screening of compounds: The National Cancer Institute Developmental Therapeutics Program (NCI DTP) diversity set of 1990 compounds ${ }^{1}$ was obtained by material transfer agreement and was diluted to a final concentration of $166.7 \mu \mathrm{M}$ in serum-containing media and placed onto cells for $72 \mathrm{hr}$ at $37^{\circ} \mathrm{C}$ and $5 \%$ $\mathrm{CO}_{2}$. A marker of cell proliferation using the substrate 5-carboxyfluorescein diacetate acetoxymethyl ester (CFDA-AM) cleaved to fluorescein by non-specific cellular esterases was used ${ }^{[19]}$. Briefly, media containing a final concentration of $5 \mu \mathrm{M}$ CFDA-AM (Molecular Probes, Eugene, OR) was added in PBS for two $h$ at $37^{\circ} \mathrm{C}$. Plates were then read by a FLUOstar Optima plate reader (BMG LABTECH, Durham, NC) using $385 \mathrm{~nm}$ excitation, $428 \mathrm{~nm}$ emission filter.

Dose-response relations of lead compounds: To 100 $\mu \mathrm{L}$ media on cells was added $100 \mu \mathrm{L}$ of a $2 \mathrm{x}(666.7$ $\mu \mathrm{M})$ stock of either doxorubicin, tetrandrine or ocresolphthalein in completed media in a single column in a 96-well plate. 1:3 dilutions were made from this highest dose by removing $66.7 \mu \mathrm{L}$ into the next column 
of cells containing $133 \mu \mathrm{L}$ media. Cells were incubated with drug for $72 \mathrm{hr}$ then the CFDA-AM assay completed as described above.

\section{Data analysis and statistics}

Drug screening: The percent inhibition of cell proliferation (as determined by CFDA-AM cleavage) as compared to untreated cells was calculated for each drug in the wells of plates. The ratio of percent inhibition of the same drug of cells grown on SISgel as compared to non-confluent monolayers also was calculated. All library compounds were screened twice for consistency.

Dose-response relations: Data were graphed as percent inhibition of proliferation as compared to untreated cells and from this $\mathrm{EC}_{50}$ values calculated using sigmoidal dose-response non-linear regression analysis (GraphPad Prism 4.0 software, San Diego, CA). Doseresponse relation data were compared to one another using two-way ANOVA with Bonferroni post-test (GraphPad Prism 4.0 software, San Diego, CA).

\section{RESULTS}

Suppression occurs in vivo: The use of GFP- or RFPlabeled cancer cells permits their fate to be followed as well as providing a convenient means to document tumor growth. Figure 1A contrasts quantifying tumor growth by calipers and by measurement of fluorescence. This shows that the two measurements show a linear relationship and that essentially similar results were obtained for actively growing tumors, no matter which method was used. Figure 1B shows the growth of tumors measured by integrated fluorescence intensity. As expected, cells that were co-injected with Matrigel uniformly formed growing tumors. In contrast, neither cells that were co-injected with saline nor those that were co-injected with SISgel formed tumors. While cells co-injected with saline generally disappeared completely within 7 days, cells co-injected with SISgel remained viable and could be seen under the skin as a small, fluorescent patch for up to 60 days postinjection.

Identification of compounds capable of inhibiting the proliferation of suppressed bladder cancer cells: It was next desired to determine whether compounds could be identified which are capable of preferentially reducing the proliferation of suppressed cancer cells using our SISgel model. For these studies, a chemical
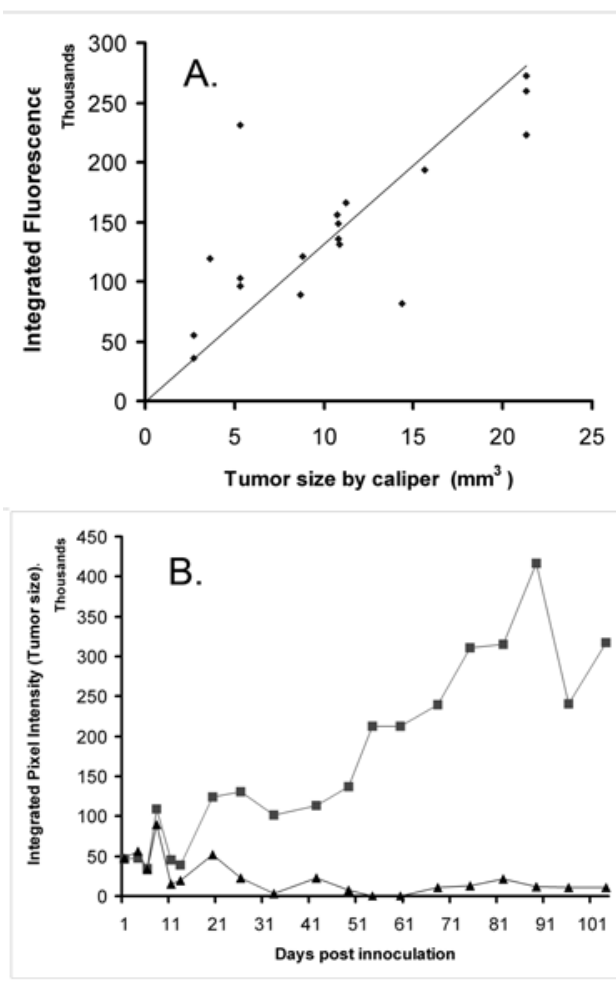

Fig. 1: Tumor growth in flank xenograft. A. Comparison of integrated fluorescence intensity as a function of tumor size estimated from calipers. B. Comparison of growth of tumors co-injected with Matrigel (ם) with those co-injected with SISgel ( $\ni$ ) as determined by integrated fluorescence intensity

Flowchart of results of chemical library screen

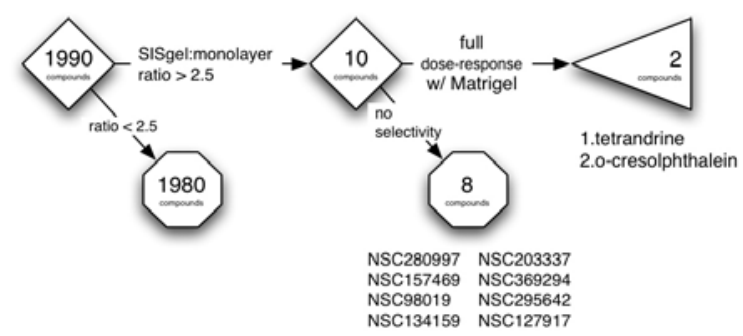

Fig. 2: Screening flowchart. J82 and TCC-SUP bladder cells on SISgel or as non-confluent monolayers in 96 well plates were initially screened with a single dose $(167.7 \mu \mathrm{M})$ of compounds from an NCI chemical library for $72 \mathrm{hr}$ then CFDA-AM added and fluorescence measured. From this, 18 compounds with activity against SISgel as compared to nonconfluent monolayers were subjected to full dose-response relations in J82 cells and 10 compounds were found to have an increased sensitivity to cells grown on SISgel as compared to Matrigel. Finally, full dose-response relations against J82, TCC-SUP and 5637 bladder cells were completed and two compounds, tetrandrine and o-cresolphthalein were found to have $\mathrm{EC}_{50}$ values significantly different in cells in SISgel as compared the same cells grown as monolayers 


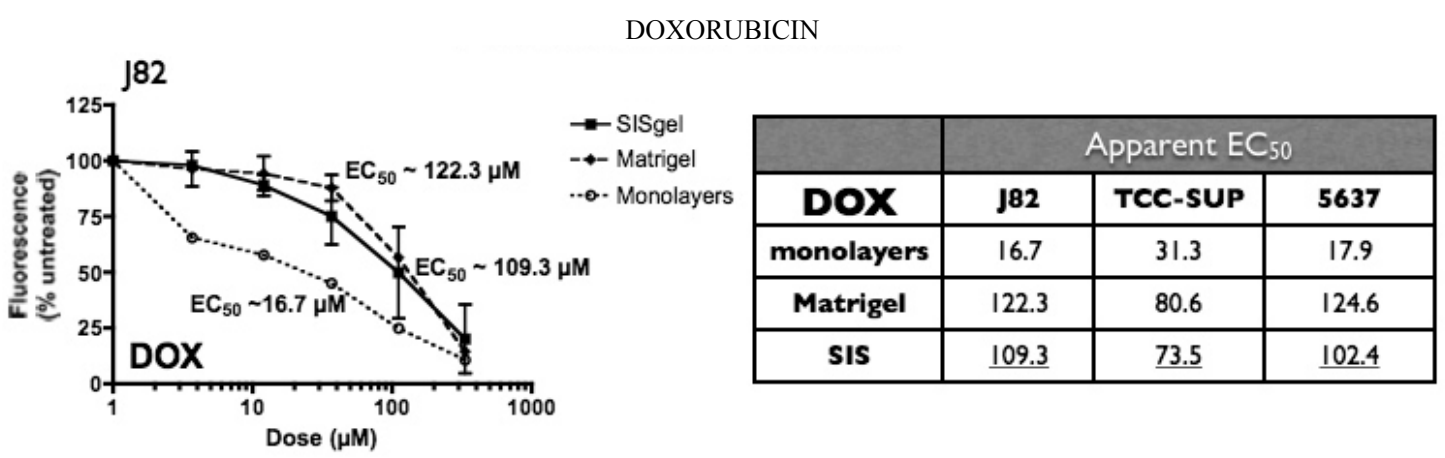

O-CRESOLOPHTHALEIN

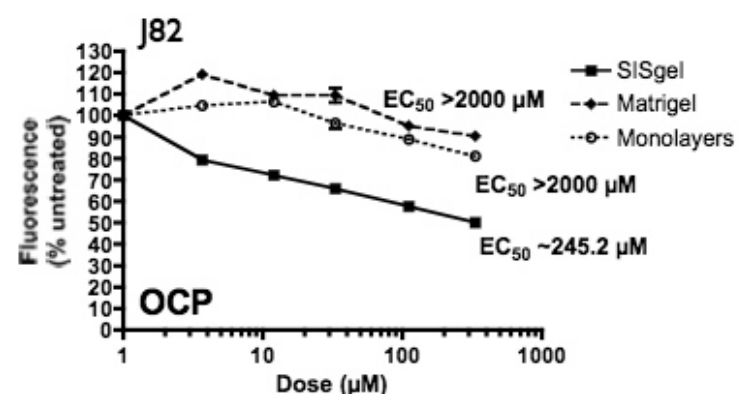

\begin{tabular}{|c|c|c|c|}
\hline & \multicolumn{3}{|c|}{ Apparent EC 50} \\
\hline OCP & $\mathbf{J 8 2}$ & TCC-SUP & $\mathbf{5 6 3 7}$ \\
\hline monolayers & $>2000$ & 1536.1 & 496.2 \\
\hline Matrigel & $>2000$ & 996.3 & 531.2 \\
\hline SIS & $\underline{245.2}$ & $\underline{198.7}$ & $\underline{146.3}$ \\
\hline
\end{tabular}

TETRANDRINE

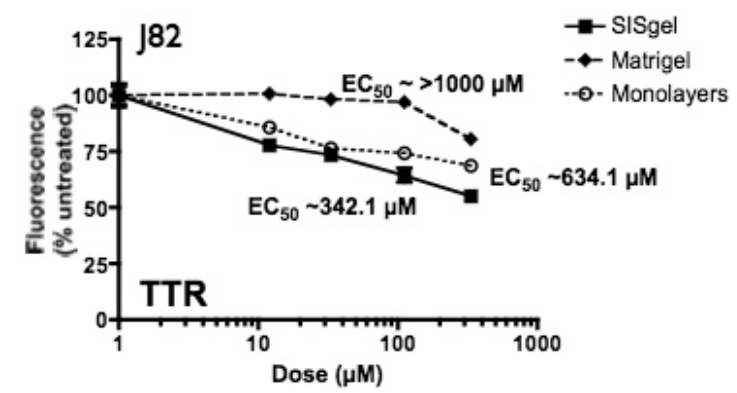

\begin{tabular}{|c|c|c|c|}
\hline & \multicolumn{3}{|c|}{ Apparent $\mathrm{EC}_{50}$} \\
\hline TTR & $\mathbf{J 8 2}$ & TCC-SUP & $\mathbf{5 6 3 7}$ \\
\hline monolayers & 1034.7 & $>1000$ & 734.1 \\
\hline Matrigel & 634.1 & 825.9 & 670.6 \\
\hline SIS & $\underline{342.1}$ & $\underline{310.3}$ & $\underline{229.1}$ \\
\hline
\end{tabular}

Fig. 3: Dose-response relations of doxorubicin (top panels), o-cresolphthalein (middle panels) and tetrandrine (bottom panels). Cells grown in the three growth environments described in the Materials and Methods section were treated with variable doses of each drug for $72 \mathrm{hr}$ then CFDA-AM added and fluorescence measured. Graphs are representative data from J82 cells and are plotted as percent control of the CFDA-AM conversion of untreated cells. The tables to the right of each graph are the $\mathrm{EC}_{50}$ data calculated for each agent in three metastatic bladder cancer cell lines - J82, TCC-SUP and 5637. Data represent the average \pm SD from at least two separate experiments with a sample size of $8-16$

library of diverse compounds was obtained through the National Cancer Institute and a single dose of $166.7 \mu \mathrm{M}$ tested for $72 \mathrm{hr}$ against J82 and TCC-SUP metastatic bladder cancer cells grown on SISgel or as nonconfluent monolayers using a 96-well microplate proliferation assay previously described ${ }^{[19]}$. This screening flowchart is shown in Fig. 2. The rationale for using non-confluent monolayers was that from our previous work $^{[19]}$, cells grown as non-confluent monolayers were the most sensitive to the traditional anticancer agents doxorubicin and mitomycin-C as compared to cells grown on either SISgel or Matrigel. Thus, any compounds found more sensitive to cells grown on SISgel as compared to non-confluent monolayers could be reasonably expected to have activity against cells grown on Matrigel. From this screen, 18 compounds found to inhibit 2.5 -fold more proliferation with cells grown in the SISgel as compared to non-confluent monolayers. These 18 compounds were termed initial "hits." 
Dose-response relations of "hits" from screen: J82 and TCC-SUP cells grown either on the normal matrix SISgel, on the tumor-derived extracellular matrix Matrigel or as non-confluent monolayers were exposed to full dose-response relations $(1.2 \mu \mathrm{M}$ to $333.3 \mu \mathrm{M})$ of these 18 compounds for $72 \mathrm{hr}$ and a proliferation assay again completed. It was found that two of the compounds, tetrandrine and o-cresolphthalein, termed final "hits," displayed at least a two-fold increase in sensitivity when cells were grown on SISgel as compared to the same cells grown on the tumor-derived matrix Matrigel or grown as non-confluent monolayers (Fig. 3, middle and bottom graphs/tables). This is in contrast to the traditional chemotherapeutic agent doxorubicin, in which cells in SISgel were less sensitive than non-confluent monolayers (Fig. 3, top graph/table). The apparent $\mathrm{EC}_{50}$ values of cells grown on SISgel as compared to on Matrigel and nonconfluent monolayers are shown in the tables to the right of the graphs in Fig. 3. It was found that both tetrandrine and o-cresolphthalein displayed significant increases in sensitivity against J82, TCC-SUP and 5637 metastatic bladder cells grown on SISgel, although the apparent $\mathrm{EC}_{50}$ values of these agents were quite high.

\section{DISCUSSION}

In the first part of this work, our SISgel model is validated as a model for suppression of the malignant phenotype. The cells in the presence of the normal extracellular matrix essentially remained alive but suppressed during the course of the experiment. These findings demonstrate that suppression occurs in vivo as well as in cell culture models and suggests the cell culture model could serve as a model system for investigation of the chemosensitivity of dormant metastatic or suppressed primary cells. In the second part of this work, a chemical library from the NCI was used as a proof-of-principle of identifying two lead compounds which target tumor cells growing in a suppressed state. These two compounds, tetrandrine and o-cresolphthalein, were found to have broad effects, showing increased sensitivity toward three metastatic bladder cancer lines. The lead compound tetrandrine has been used as a chemosensitizing agent to overcome multidrug resistance in cancer ${ }^{[20]}$ by blocking the efflux of drugs from energy-driven pumps such as Pglycoprotein $^{[21]}$. Further, tetrandrine has been shown to induce apoptosis in cancer cells ${ }^{[22]}$. Thus, this agent could be given sequentially with traditional cytotoxic agents to increase their efficacy as well as to attack suppressed cells. The second lead compound, o- cresolphthalein is a calcium complexing agent used as a colorimetric assay to determine calcium levels ${ }^{[23]}$. As such, this agent might be altering calcium-mediated signaling and or cell adhesion mechanisms to result in decreased cell proliferation.

Agents developed against dormant tumors or micrometastases would probably be administered as maintenance therapy after resection, chemotherapy, or radiation of the primary tumor. Thus it is imperative that these agents have systemic (i.e., whole-body) efficacy to target occult dormant cells or micrometastases, low cumulative toxicity because these agents will be used long-term, and oral bioavailability to ensure compliance. The current approach to the treatment of micrometastases and dormant tumors tends toward using traditional therapeutic strategies as additional "maintenance therapy" to existing neoadjuvant therapy ${ }^{[24]}$. While a cocktail of conventional drugs, radiation or surgery can oftentimes successfully eliminate the primary tumor, these interventions have not proven useful in eliminating dormant tumor cells ${ }^{[25,26]}$ or micrometastases ${ }^{[24]}$. In addition, by the time the patient is diagnosed with cancer, the disease has oftentimes advanced to the stage of micrometastases and dormant tumors in sentinel lymph nodes and secondary sites, again leading to a worse prognosis $^{[27]}$. Thus, there is a need for research to be focused on the discovery and development of therapeutic agents that selectively kill the dormant tumor cells and micrometastases.

Our lead compounds can be modified chemically to study their structure-activity relationships (SAR) and to design compounds with better potency with the retention or improvement of the selectivity for killing suppressed cells. Moreover, combination studies of these drugs together with conventional drugs may shed light on a possible additive, synergistic, or in the case of tetrandrine, chemosensitizing effect that will help in designing regimens that target recurrences and micrometastases. The process of "bench to bedside" is a long and arduous one which, of late, has been largely disappointing in terms of truly new drugs ${ }^{[28]}$. However, altering drug screening from the traditional "brute force" approach using 2-dimensional lawns of cells grown on plastic to a more thoughtful screening with cells grown in 3-dimensions on appropriate matrices may provide more relevant lead compounds.

\section{ACKNOWLEDGEMENTS}

This work was funded by DK 069808 (REH), start up funds (M.A.I.) and by a NIH/NCRR Center for 
Biomedical Research Excellence program project "Mentoring Vision Research in Oklahoma" (1P20RR017703-01) to M.A.I. (promising junior investigator). Satyendra Shenoy is a visiting scholar being supported using unrestricted funds Nicolas Piramal. Nicolas Piramal did not provide any formal support for these studies.

Abbreviations: DOX, doxorubicin; ECM, extracellular matrix; MMC, mitomycin C; SIS, small intestine submucosa.

\section{REFERENCES}

1. Jemal, A., T. Murray, E. Ward, A. Samuels, R.C. Tiwari, A. Ghafoor, E.J. Feuer and M.J. Thun, 2005. Cancer statistics, 2005. CA Cancer J. Clin., 55: $10-30$.

2. Cohen, S.M., T. Shirai and G. Steineck, 2000. Epidemiology and etiology of premalignant and malignant urothelial changes. Scand. J. Urol. Nephrol. Suppl., pp:105-115.

3. Amling, C.L., 2001. Diagnosis and management of superficial bladder cancer. Curr. Probl. Cancer, 25: 219-278.

4. Heney, N.M., S. Ahmed, M.J. Flanagan, W. Frable, M.P. Corder, M.D. Hafermann and I.R. Hawkins, 1983. Superficial bladder cancer: Progression and recurrence. J. Urology, 130: 1083-1086.

5. Bellmunt, J., R. de Wit, S. Albiol, J. Tabernero, J. Albanell and J. Baselga, 2003. New drugs and new approaches in metastatic bladder cancer. Crit. Rev. Oncol. Hematol., 47: 195-206.

6. Herr, H.W. and S.M. Donat, 2001. Outcome of patients with grossly node positive bladder cancer after pelvic lymph node dissection and radical cystectomy. J. Urology, 165: 62-64.

7. Pycha, A., C. Mian, J. Hofbauer, A. Haitel, H. Wiener and M. Marberger. 1998. Does topical instillation therapy influence chromosomal aberrations in superficial bladder cancer? J. Urology, 159: 265-269.

8. Sonpavde, G. and D.P. Petrylak, 2006. Perioperative chemotherapy for bladder cancer. Crit. Rev. Oncol. Hematol., 57:133-144.

9. Grossman, H.B., R.B. Natale, C.M. Tangen, V.O. Speights, N.J. Vogelzang, D.L. Trump, R.W. Vere White, M.F. Sarosdy, D.P. Wood, Jr., D. Raghavan and E.D. Crawford. 2003. Neoadjuvant chemotherapy plus cystectomy compared with cystectomy alone for locally advanced bladder cancer. New England J. Med., 349: 859-866.

10. Jocham, D., F. Witjes, S. Wagner, B. Zeylemaker, van Moorselaar J., M.O. Grimm, R. Muschter, G. Popken, F. Konig, R. Knuchel and K.H. Kurth, 2005. Improved detection and treatment of bladder cancer using hexaminolevulinate imaging: a prospective, phase III multicenter study. J. Urology, 174: 862-866.

11. Duggan, B.J., S.B. Gray, J.J. McKnight, C.J. Watson, S.R. Johnston and K.E. Williamson, 2004. Oligoclonality in bladder cancer: The implication for molecular therapies. J. Urology, 171: 419-425.

12. Linn, J.F., M. Lango, S. Halachmi, M.P. Schoenberg and D. Sidransky, 1997. Microsatellite analysis and telomerase activity in archived tissue and urine samples of bladder cancer patients. Intl. J. Cancer, 74: 625-629.

13. Steiner, G., M.P. Schoenberg, J.F. Linn, L. Mao and D. Sidransky, 1997. Detection of bladder cancer recurrence by microsatellite analysis of urine. Nature Medicine, 3: 621-624.

14. Rao, J.Y., G.P. Hemstreet, R.E. Hurst, R.B. Bonner, P.L. Jones, K.W. Min and Y. Fradet, 1993. Alterations in phenotypic biochemical markers in bladder epithelium during tumorigenesis. Proc. Natl. Acad. Sci. USA, 90: 8287-8291.

15. Czerniak, B., V. Chaturvedi, L. Li, S. Hodges, D. Johnston, J.Y. Roy, R. Luthra, C. Logothetis, A.C. von Eschenbach, H.B. Grossman, W.F. Benedict and J.G. Batsakis, 1999. Superimposed histologic and genetic mapping of chromosome 9 in progression of human urinary bladder neoplasia: implications for a genetic model of multistep urothelial carcinogenesis and early detection of urinary bladder cancer. Oncogene, 18: 1185-1196.

16. Stoehr, R., S. Zietz, M. Burger, T. Filbeck, S. Denzinger, E.C. Obermann, C. Hammerschmied, W.F. Wieland, R. Knuechel and A. Hartmann, 2005. Deletions of chromosomes 9 and $8 p$ in histologically normal urothelium of patients with bladder cancer. Eur. Urology, 47: 58-63.

17. Hurst, R.E., K.D. Kyker, R.B. Bonner, R.G. Bowditch and G.P. Hemstreet, 2003. Matrixdependent plasticity of the malignant phenotype of bladder cancer cells. Anticancer Res., 23: 31193128.

18. Kyker, K.D., D.J. Culkin and R.E. Hurst. 2003. A model for 3-dimensional growth of bladder cancers to study mechanisms of phenotypic expression. Urologic Oncol., 21: 255-261.

19. Hurst, R.E., C.D. Kamat, K.D. Kyker, D.E. Green and M.A. Ihnat, 2005. A novel multidrug resistance 
phenotype of bladder tumor cells grown on Matrigel or SIS gel. Cancer Lett., 217: 171-180.

20. Zhu, X., M. Sui and W. Fan, 2005. In vitro and in vivo characterizations of tetrandrine on the reversal of P-glycoprotein-mediated drug resistance to paclitaxel. Anticancer Res., 25: 1953-1962.

21. Fu, L.W., Y.M. Zhang, Y.J. Liang, X.P. Yang and Q.C. Pan, 2002. The multidrug resistance of tumour cells was reversed by tetrandrine in vitro and in xenografts derived from human breast adenocarcinoma MCF-7/adr cells. Eur. J. Cancer, 38: 418-426.

22. Lai, Y.L., Y.J. Chen, T.Y. Wu, S.Y. Wang, K.H. Chang, C.H. Chung and M.L. Chen, 1998. Induction of apoptosis in human leukemic U937 cells by tetrandrine. Anticancer Drugs, 9: 77-81.

23. Morin, L.G., 1974. Direct colorimetric determination of serum calcium with ocresolphthalein complexon. Am. J. Clin. Pathol., 61: 114-117.

24. Epstein, R.J., 2005. Maintenance therapy to suppress micrometastasis: the new challenge for adjuvant cancer treatment. Clin. Cancer Res., 11: 5337-5341.
25. Ranganathan, A.C., A.P. Adam, L. Zhang and J.A. guirre-Ghiso, 2006. Tumor cell dormancy induced by p38SAPK and ER-stress signaling: an adaptive advantage for metastatic cells? Cancer Biol. Ther., 5: 729-735.

26. Braun, S. and K. Pantel, 1999. Micrometastatic bone marrow involvement: detection and prognostic significance. Med. Oncol., 16: 154-165.

27. Leong, S.P., B. Cady, D.M. Jablons, J. GarciaAguilar, D. Reintgen, J. Jakub, S. Pendas, L. Duhaime, R. Cassell, M. Gardner, R. Giuliano, V. Archie, D. Calvin, L. Mensha, S. Shivers, C. Cox, J.A. Werner, Y. Kitagawa and M. Kitajima, 2006. Clinical patterns of metastasis. Cancer \& Metastasis Rev., 25: 221-232.

28. Cuatrecasas, P., 2006. Drug discovery in jeopardy. J. Clin. Invest., 116: 2837-2842. 